\title{
Discussion on the Development Mode of Medical Health Management against the "Internet +" Background
}

\author{
Haibin Wu, Xiaorong Song, Bomin Chen, Jiwei Lin, Jingwei Li, Maoqing Tan, \\ Bohan Li and Dongcai Wang*
}

Shenzhen TCM Hospital, Shenzhen 518033, China.

*13823713909@163.com

Keywords: Internet +; Health management; Application.

\begin{abstract}
Internet + " health management can achieve real-time data acquisition, intelligent monitoring and other functions through applying intelligent mobile phone, smart wearable, cloud computing, big data, artificial intelligence and other advanced technology comprehensively, which will become a new medical service mode in the future. This paper analyzed the role, the situation, the trend and the technology progress of "internet +" health management. Simultaneously, an example of this mode was given to illustrate the build and implement of "internet +" health management.
\end{abstract}

\section{Introduction}

Modern medical studies have shown that many diseases are not caused by biological factors, but are caused by bad lifestyle, psychological and environmental factors. This view of disease is called "biological psycho social medical model", and its main clinical measure is to implement health management [1]. At the end of the 1950s, the United States took the lead in health management. With the continuous enrichment and development of actual business content, it was proved to reduce the risk of illness and reduce medical expenses. At home, health management is in its infancy, but because of the rapid development of high technology, such as network technology, sensing technology, mobile Internet and wearable equipment, large data and so on, the health management is constantly expanding in health management [2]. Therefore, health management in China is extremely likely to achieve curved road overtaking, "Internet + health management" as a new type of social health care system, this mode will usher in blowout development and bring new services to people's health.

\section{The Development Trend of "Internet + Health Management" Mode}

The traditional health management technology is lagging behind, the acquisition, processing and application of information is very limited. Medical services are restricted by multiple factors such as time, space, technology, medical resources and funds. Modern health management is an organic combination of advanced technology and modern medical technology such as information technology, sensing technology, large data and cloud services, and a set of perfect and personalized service system based on preventive health care and clinical medicine. The purpose is to collect, analyze and deal with the health data in real time. To provide guidance for prevention, intervention or treatment, and to establish a health guarantee system for healthy people, especially subhealthy people, to effectively reduce the incidence of related diseases, as shown in Fig 1.

In the future, "Internet + health management" combines the high and new technologies such as cloud computing, Internet of things and big data with modern medical rescue and preventive health care system [3] to make full use of the optimization and integration of the Internet in medical treatment. Management, sleep management and even diet management completely eliminate the limitations of the traditional physical and nursing service system, which are limited by time and space. It can form a more extensive medical health guarantee and management format with the Internet as the infrastructure and service equipment and provide the necessary information for the information of health management in an all-round way. Support. The introduction of "Internet + health management" can provide a more professional, systematic and personalized health education and 
guidance to the healthy population, provide a comprehensive and scientific health management service model [4], and extend the hospital services to the outside of the hospital. Through regular health assessment, real-time monitoring and data analysis, the needs are given. All kinds of information and interventions can reduce risks and really change the traditional health management mode.

\section{( Health)}

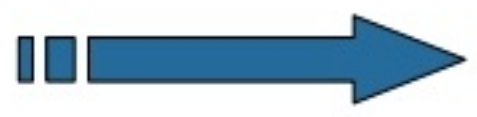

(Death)

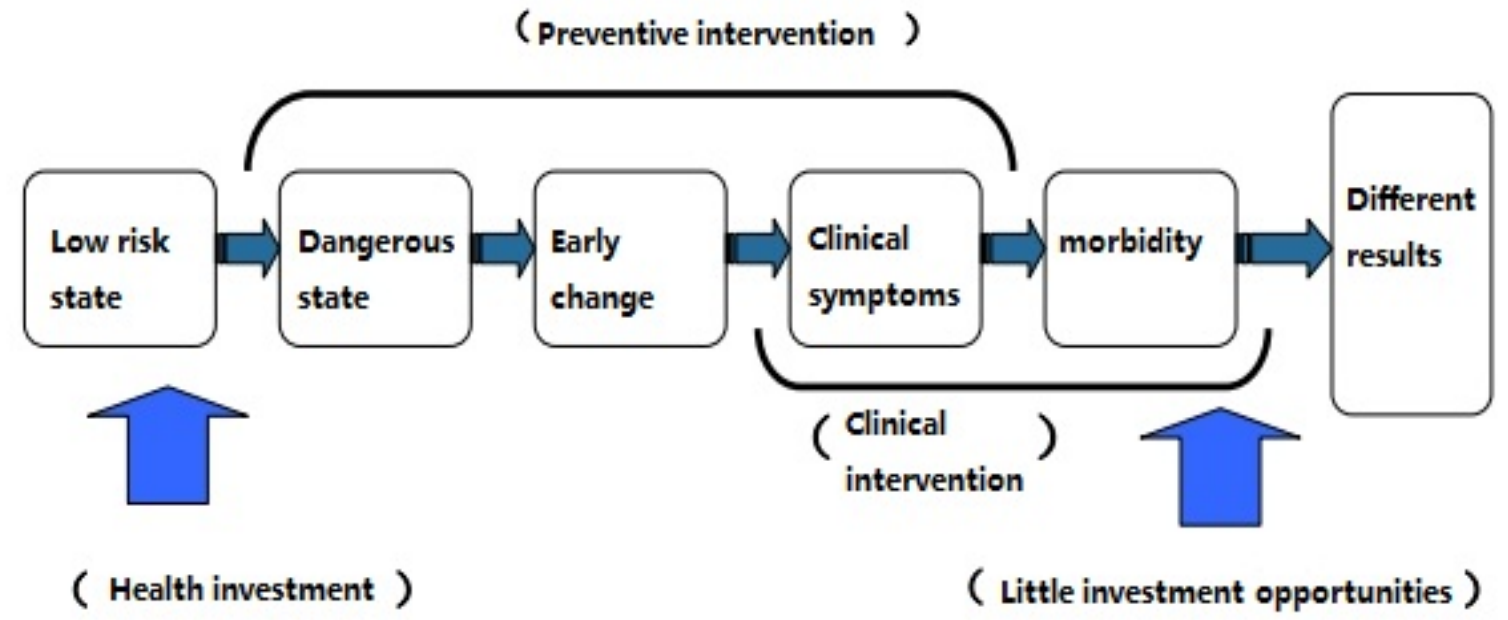

Fig 1. Health management: disease prevention early, early treatment for the disease, combining prevention with control

\section{The Role of "Internet + Health Management" Mode}

With the rapid development of China's economy, social economic structure and lifestyle changes, people's health awareness has also changed, healthy consumption demand is growing, and gradually from a single medical treatment type to disease prevention, health care and health promotion type [5].

According to the economic model, we can see the important relationship between economic growth and health, as shown in Fig. 2. In the west, health management plans can effectively reduce personal risk and reduce medical expenses. Health management in the United States plays an active role in prevention and intervention, thereby reducing the overall risk. Today, health management based on "Internet +" can detect and evaluate the risk factors of disease through systematic detection and evaluation. Through health education to individuals or groups, people can learn self-management and daily health care and improve their awareness and level of self-management health, especially the health risk related to lifestyle. Factors to assess and monitor, provide personalized intervention, greatly reduce the risk of disease, and thus successfully block, delay, even reverse the occurrence and development of the disease, to achieve the purpose of maintaining health.

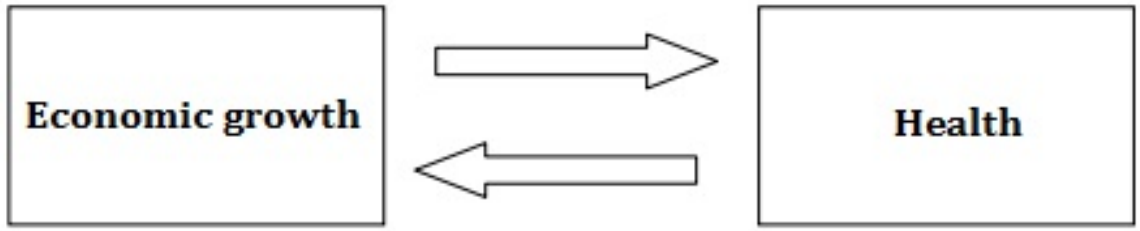

Fig 2. The relationship between economic growth and health

\section{The Main Technology of "Internet + Health Management" Mode}

\subsection{Smart Phone Technology in Health Management.}

The application of smart phones in health management, such as the "pocket fitness coach" App (run keeper), can call the GPS global positioning function of the smartphone to record the user's running route, speed, time, calorie, heart rate. There's a Gudong mobile App, which uses a rich sports 
challenge to encourage the manager to stick to his own training program. The health book in the apple cell phone has a very rich health function for blood sugar, blood pressure, heart rate and even sleep monitoring. The application of smart watches and bracelets in health monitoring, such as Samsung Gear2 smart watches, has some functions in sports and health management.

\subsection{Intelligent Wearable Technology in Health Management.}

Apple, Google and Samsung all want to add the function of monitoring blood sugar in wearable devices to help diabetics monitor blood sugar effectively. For example, Google and Nuo Hua (Novartis) have agreed to use contact lenses to detect glucose levels in tears. Samsung has released a new hardware product design Simband health wristband, which is described by Samsung as a new modular wearable health equipment reference platform. Associated with the SAM I system, it is a cloud computing service device that can track blood pressure, respiration, heart rate variability and body surface temperature throughout the day. Playtabase has launched a home automation system, Reemo, which helps users to control Internet devices through simple gestures that can help the elderly, be ill and care for the disabled, and reduce their dependence on others. The US Food and drug administration allows two sports recording watches (MotionWatch and Pro-Diary) are used in the field of clinical trials, which is another government subsidy program in wearable technology after WellDoc's mobile phone APP monitors the diabetes project as a government subsidy program. The extensive use of wearable technology in the field of health care will prompt pharmaceutical companies to transform from product-based production mode to service user mode.

\subsection{Cloud Computing and Large Data Technology in Health Management.}

The application of cloud computing in health management can effectively solve the problems of inadequate medical resources, uneven distribution, subhealth and chronic diseases, aging medical care, low utilization of public health resources, and the establishment of a personal health management system, which combines cloud computing and intelligent mobile terminals (such as mobile phones). A case database was established to monitor the patient's health in real time on the basis of the patient's symptoms and signs and combined with the large data analysis technique [6] to give the patient a possible prediction of the disease in advance, to solve the medical difficulties in the remote rural areas and to ignore the timely treatment of some diseases because of the poverty and so on, and the city, as well as the city. A large number of sub-health people are too busy to attend to the potential problems of personal health because of their busy work. In addition, the application of Internet + based cloud computing and large data technology in health management has broad prospects for real-time collection of patients' pathological data, closed loop tracking and monitoring, difficult and difficult disease analysis and diagnosis, infectious disease prediction, health and public health emergency treatment.

\section{Typical Application of "Internet + Health Management" Mode}

\subsection{Application of Real Time Health Data Acquisition.}

Through the application of the "Twelve basket health management" model to the large data, the traditional way of the traditional doctor's face diagnosis is solved. The user can automatically send the corresponding management scheme for the user through the record of its own health data, and the user's initiative can be maintained through a series of intelligent services and interactive community design. Making continuous health management a feasible and popular mode of health management. Through the intervention of the "Internet +" new technology, most people develop a self-management awareness of health, and change their health behavior with the health information derived from the Internet, look for a healthy lifestyle [7], and reduce the incidence of disease.

\subsection{Application in Telemedicine Service.}

The application of the remote video diagnosis and treatment service system based on the Internet has relieved the difficult problems in the remote areas and the grass-roots level, so that the patients can see the experts at home. At the same time, it also improves the diagnosis and treatment skills of the doctor's difficult and miscellaneous diseases at the grass-roots level, and also relieves the imbalance of medical resources. In addition, based on 3G, 4G and even future 5G and wireless broadband access technology, the network of wireless remote monitoring blood sugar has been 
established to realize remote diagnosis and treatment of diabetes [8]. It provides convenient and quick medical treatment for diabetics and designs a wireless remote blood glucose monitor for monitoring individual and community multi person blood glucose conditions. Protect the network system. The successful application of the remote diabetic retinopathy image diagnosis system provides the basic medical workers with the opportunity to learn and recognize the diabetic retinopathy, and make the basic medical personnel master the diagnosis and treatment technology in an all-round way, reduce the incidence of the complications and the existence of missed diagnosis, mistaken diagnosis and mistaken treatment. It is the treatment of patients with diabetic retinopathy.

\section{Summary}

The "Internet + health management" model is the application of intelligent wearable technology, cloud computing and large data to human health management. It realizes the collection, processing, transmission and sharing of health data. It solves the problem of the information island and the shortage of high-quality medical resources in the traditional health management. To a great extent, it is changing. Even subverting the traditional health management model is speeding up the construction of a new medical health management system, which transforms the patient's needs from the traditional hospital centered and disease treatment based medical model to the modern health management model, which is based on prevention, remote, shared and real time tracking.

\section{Acknowledgments}

Fund: Sanming Project of Medicine in Shenzhen(SZSM201612081).

\section{References}

[1]. Zeng Xuemei, Prince Yue. Clinical research of "bio psycho social" medical model Application. Psychological technology and application, 2014 (11): 36-38.

[2]. Li Haiyang moves towards mobile Internet, innovating health management service mode. Chinese digital medicine, 2014, 12 (9): 62.

[3]. Chen Yiyi, Qiu Yunqing, Zhou Min, et al. First aid medical information association based on mobile cloud platform design. Chinese hospital management, 2014, 34 (7): 52-54.

[4]. Liu Zi Ling. Construction of wireless mobile medical big data health management platform. Chinese medical equipment, 2016 (3): 105-107.

[5]. Yu dlin, Gao Lei, Sun Jinhai, etc. The application of large data technology in health management. The Journal of hospital management of the PLA, 2016, 23 (1): 44-48.

[6]. Yang Bin, Hua Zexi, Yang Guanjian. Personal health smart tube based on cloud computingSystem. Modern computer (Professional Edition), 2014 (12): 69-74.

[7]. Chen Yifan, Hui Ling, Xue Wanjun, and so on. Internet health of Hefei residents Information utilization, health concept and behaviour investigation. Chinese Journal of social medicine, 2015, 32 (3): 183-185.

[8]. Wu Qiong, Chen Min. Application of remote outpatient system based on visual networking. Chinese hospital management, 2015, 35 (4): 50-52. 